\title{
Amalgamation of Metamaterial and SIW Technologies for Realizing Wide-Bandwidth and High-Radiation Properties of On-Chip Antennas for Application in Packaging of Terahertz Components
}

\author{
Mohammad Alibakhshikenari ${ }^{1 *}$, Bal S. Virdee ${ }^{2}$, Mohsen Khalily ${ }^{3}$, Chan H. See ${ }^{4,5}$, Sam Jeffery Fishlock ${ }^{6}$, Navneet
} Soin $^{6}$, James McLaughlin ${ }^{6}$, Raed A. Abd-Alhameed ${ }^{7,8}$, and Ernesto Limiti ${ }^{1}$

\author{
${ }^{1}$ Electronic Engineering Department, University of Rome "Tor Vergata", Via del Politecnico 1, 00133, Rome, ITALY \\ ${ }^{2}$ London Metropolitan University, Center for Communications Technology \& Mathematics, School of Computing \& Digital Media, \\ London N7 8DB, UK \\ ${ }^{3}$ Institute for Communication Systems, Home of 5G Innovation Centre (5GIC), University of Surrey, Guildford, GU2 7XH, UK \\ ${ }^{4}$ School of Engineering, University of Bolton, Deane Road, Bolton, BL3 5AB, UK \\ ${ }^{5}$ School of Eng. \& the Built Environment, Edinburgh Napier University, 10 Colinton Rd., Edinburgh, EH10 5DT, UK \\ ${ }^{6}$ School of Engineering, Nanotechnology and Integrated Bioengineering Centre (NIBEC), University of Ulster, Newtownabbey, \\ BT37 0QB, Northern Ireland, UK \\ ${ }^{7}$ School of Electrical Engineering \& Computer Science, University of Bradford, Bradford, BD7 1DP, UK
}

*alibakhshikenari@ing.uniroma2.it

\begin{abstract}
This paper shows that by employing a combination of metamaterial (MTM) and substrate integrated waveguide (SIW) technologies, the drawbacks of narrow-bandwidth and low radiation properties encountered in terahertz on-chip antennas can be overcome. In addition, an effective feeding mechanism is introduced to excite the on-chip antenna. The proposed antenna is constructed on the three stacked layers comprising Silicon-metal-Silicon substrates. Dimensions of on-chip antenna are $1 \times 1 \times 0.265 \mathrm{~mm}^{3}$. The on-chip antenna is shown to have an average impedance match, gain, and efficiency parameters of $-35 \mathrm{~dB}, 8.5 \mathrm{dBi}$, and $67.5 \%$, respectively, over a wide frequency range of $0.20-0.22 \mathrm{THz}$.
\end{abstract}

Keywords: On-chip antenna, metamaterial, substrate integrated waveguide, terahertz, wideband, high-efficiency, silicon, feeding mechanism.

\section{INTRODUCTION}

The interest in terahertz band $(0.1-10 \mathrm{THz})$ is steadily growing as this band enables several important applications such as biomedical imaging, ultrafast wireless communication, remote non-destructive inspection of packaged goods, and security screening. The current terahertz systems are of low power and low sensitivity. This necessitates the development of terahertz antennas for on-chip applications. Several CMOS on-chip antennas have been studied and manufactured to meet the demand of terahertz radio on-chip front-end circuit systems. However, most of the terahertz on-chip antennas have problems of low radiation efficiency and narrow bandwidth, which are caused by increased losses from substrate and conductors and very thin thickness of substrate between antenna and ground plane. Standard rectangular CMOS on-chip patch antennas have shown fractional bandwidth and radiation efficiency less than $10 \%[1,2]$.
In this paper metamaterial (MTM) and substrate integrated waveguide (SIW) technologies are used to overcome the restrictive bandwidth, gain and efficiency limitations for small antennas [3-5]. A combination of these two technologies are used to design low-cost and high-performance terahertz integrated-circuit antennas for on-chip applications. The benefit derived from metamaterial technology is to enhance the bandwidth and radiation performances without compromising the total size of the antenna.

\section{High PeRfPRmances ON-ChIP ANTENNA}

To realize a high-performance antenna in terms of frequency range, radiation gain and efficiency from a highly compact on-chip area is very challenging due to the small effective aperture area of the chip as well as adverse effects of surface waves and substrate loss. To solve these drawbacks, we have employed metamaterial and substrate integrated waveguide technologies. In addition, a novel feeding mechanism is employed, which is based on an open-ended microstrip-line, to excite the antenna.

The proposed on-chip antenna was constructed on three stacked layers comprising two outer layers made of Silicon substrate that sandwiches a ground-plane layer made of Aluminium. The Silicon layer has a thickness of $125 \mu \mathrm{m}$, dielectric constant of 11.9, and loss-tangent of 0.00025 . The Aluminium layer has a thickness of $5 \mu \mathrm{m}$.

In initial step before constructing the stack was to implement metamaterial properties in the ground-plane. This involved embedding numerous circular slots in the ground-plane to minimise substrate loss, suppress the propagation of surface waves, and to couple electromagnetic energy from the bottom Silicon layer to the radiation patch, which is realized on the top Silicon layer. Fig.1 shows the ground-plane (GND) with embedded circular slots. The effective aperture area of the antenna is increased by simply embedding the circular slots 
in the ground-plane. The dimensions of the slots are identical. The slots essentially act like series left-handed (LH) capacitances $\left(C_{L}\right)$.

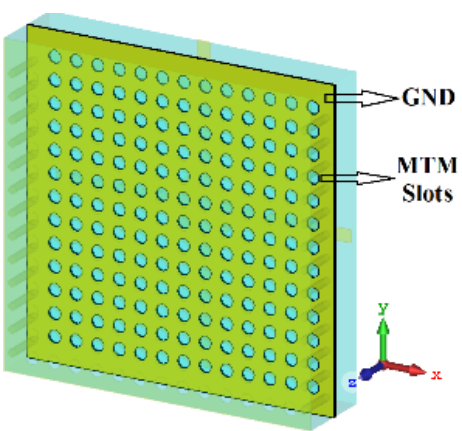

(a) Top view

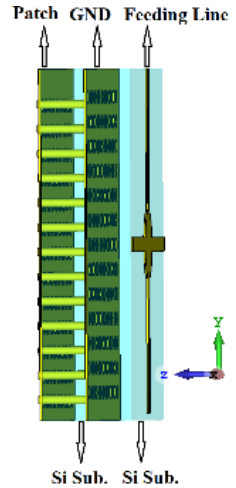

(b) Side view
Fig.1. View of the ground plane (GND) sandwiched between two Silicon substrates (Si Sub.) of the proposed on-chip antenna.

In the second step, square radiation patches are implemented on the top Silicon layer, which is then placed on the middle ground-plane layer.

Surface waves and substrate loss was reduced by incorporating metallic via-holes on both sides of the antenna to create a wall analogous to a substrate integrated waveguide. The metallic via-holes connect the radiation patch to the ground-plane by passing through the top Silicon layer. The metallic via-holes act like a shunt $\mathrm{LH}$ inductance $\left(L_{L}\right)$. The combination of series $C_{L}$ and shunt $L_{L}$ transform the structure to exhibit metamaterial properties $[3,4]$. Fig. 2 shows the view of the on-chip antenna with MTM slots and SIW vias. The results below show improvement in the antenna's performance using this approach.

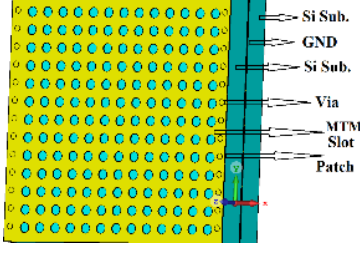

(a) Top view

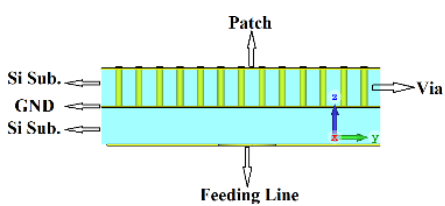

(b) Side view
Fig.2. View of the proposed on-chip antenna implemented using MTM slots and SIW metallic via-holes.

In the final step, a novel feeding structure is employed to excite the proposed antenna structure. The feeding structure is composed of a cross-shaped microstrip-line with a circular central joint to improve matching with the input port. This structure, shown in Fig. 3, is implemented on the bottom side of the lower Silicon layer, which is under the ground-plane layer, as shown in Fig. 2. Three branches of the feed structure are open-ended microstriplines and excitation is via the fourth line using coplanar waveguide (CPW). Electromagnetic energy from the $\mathrm{CPW}$ port excites the feeding structure's three tentacles to couple it to the radiation patches on the top Silicon layer via the circular slots in the ground-plane. The results below confirm improvement in frequency range of operation and radiation properties of the on-chip antenna.

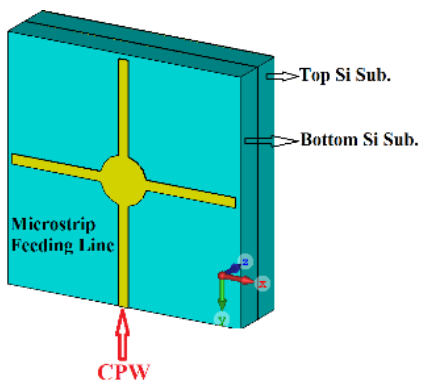

Fig.3. Microstrip feeding structure implemented on the bottom side of the lower Silicon layer.

The structural parameters of the on-chip antenna are tabulated in Table I. The reflection coefficient, radiation gain and efficiency performance across the antenna's operating frequency range are shown in Fig.4. These results confirm the proposed on-chip antenna is capable of operating from $0.2-0.22 \mathrm{THz}$ for $\mathrm{S}_{11}<-15 \mathrm{~dB}$ with average impedance matching, gain and efficiency of $-35 \mathrm{~dB}, 8.5 \mathrm{dBi}$ and $67.50 \%$, respectively. In addition, the proposed antenna is easy to design and implement.

\section{TABLE I. STRUCTURAL PARAMETERS}

\begin{tabular}{|c|c|}
\hline Size of Antenna & $1000 \times 1000 \times 265 \mu \mathrm{m}^{3}$ \\
\hline Size of GND & $1000 \times 1000 \times 5 \mu \mathrm{m}^{3}$ \\
\hline Thickness of Silicon & $125 \mu \mathrm{m}$ \\
\hline Diameter of Slot & $20 \mu \mathrm{m}$ \\
\hline Height of Via & $135 \mu \mathrm{m}$ \\
\hline Diameter of Via & $12 \mu \mathrm{m}$ \\
\hline Width of Fed Line & $40 \mu \mathrm{m}$ \\
\hline Length of Fed Line & $400 \mu \mathrm{m}$ \\
\hline Diameter of Fed Line & $200 \mu \mathrm{m}$ \\
\hline
\end{tabular}

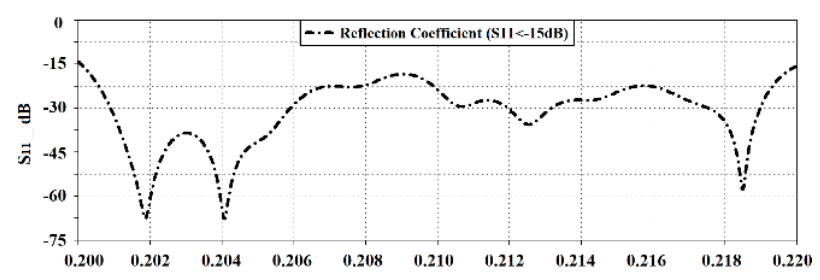

Frequency_TH,

(a) Reflection coefficient response $\left(\mathrm{S}_{11}<-15 \mathrm{~dB}\right)$.

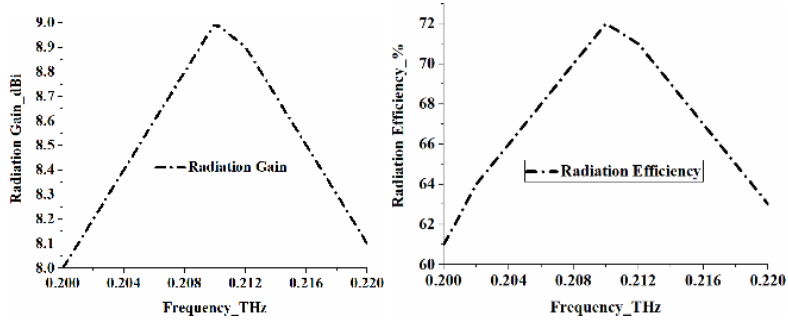

(b) Gain and efficiency as a function of frequency.

Fig.4. Performance parameters of the proposed on-chip antenna.

\section{CONCLUSION}

Feasibility of an on-chip antenna design is presented for a wideband, high-gain and high-efficiency terahertz 
integrated-circuit applications. The antenna design is based on using metamaterial and substrate integrated waveguide technologies implemented on Silicon substrate. The antenna is excited using a novel open-circuited structure fed via coplanar waveguide port.

\section{ACKNOWLEDGEMENTS}

This work is partially supported by innovation programme under grant agreement H2020-MSCA-ITN-2016 SECRET-722424 and the financial support from the UK Engineering and Physical Sciences Research Council (EPSRC) under grant EP/E022936/1.

\section{REFERENCES}

[1] Hua Zhu, Nan Li, Junjie Zeng, Xiuping Li, Bo Ai, “A Novel 0.22-THz On-chip Antenna Based AMCs", Asia-Pacific Microwave Conference 2014, pp.366-368, 4-7 Nov. 2014, Sendai, Japan.

[2] Ahmed Shehata Abdellatif, Aidin Taeb, Safieddin Safavi-Naeini, Klaus Schmalz, "A Terahertz High-efficiency On-Chip Antenna", 2014 IEEE Antennas and Propagation Society International Symposium, pp. 1485-14.86, 6-11 July 2014, Memphis, TN, USA.

[3] M. Alibakhshikenari, B. S. Virdee, A. Ali, and E. Limiti, "Extended Aperture Miniature Antenna Based on CRLH Metamaterials for Wireless Communication Systems Operating Over UHF to C-Band" Radio Science, Vol.53, Iss.2, Feb. 2018, pp.154-165.

[4] M. Alibakhshikenari, Bal S. Virdee, Abdul Ali, and Ernesto Limiti, "Miniaturized Planar-Patch Antenna Based on Metamaterial L-shaped Unit-Cells for Broadband Portable Microwave Devices and Multiband Wireless Communication Systems" IET Microwaves, Antennas \& Propagation, Volume 12, Issue 7, 13 June 2018, p. 1080 - 1086.

[5] K. Wu, Y. J. Cheng, T. Djerafi, and W. Hong, "Substrate-Integrated Millimeter-Wave and Terahertz Antenna Technology", Proceedings of the IEEE, vol. 100, No. 7, July 2012, pp. 2219-2232. 